\section{BMJ Paediatrics Open}

\title{
Extreme neonatal hyperbilirubinaemia in refugee and migrant populations: retrospective cohort
}

Eva Maria Nadine Wouda (D , , ${ }^{1,2}$ Laurence Thielemans, ${ }^{1,3}$ Mue Chae Darakamon, ${ }^{1}$ Aye Aye Nge, ${ }^{1}$ Wah Say, ${ }^{1}$ Sanda Khing, ${ }^{1}$ Borimas Hanboonkunupakarn, ${ }^{4}$ Thatsanun Ngerseng, ${ }^{4}$ Jordi Landier, ${ }^{1,5}$ Patrick Ferry van Rheenen, ${ }^{6}$ Claudia Turner, ${ }^{4,7,8}$ Francois Nosten, ${ }^{1,8}$ Rose McGready, ${ }^{1,8}$ Verena llona Carrara (1) 1,8,9

\section{ABSTRACT}

Objective To describe neonatal survival and long-term neurological outcome in neonatal hyperbilirubinaemia $(\mathrm{NH})$ with extreme serum bilirubin (SBR) values.

Design Retrospective chart review, a one-off neurodevelopmental evaluation.

Setting Special care baby unit in a refugee camp and clinics for migrant populations at the Thailand-Myanmar border with phototherapy facilities but limited access to exchange transfusion (ET).

Patients Neonates $\geq 28$ weeks of gestational age with extreme SBR values and/or acute neurological symptoms, neurodevelopment evaluation conducted at 23-97 months of age.

Main outcome measures Neonatal mortality rate, prevalence of acute bilirubin encephalopathy (ABE) signs, prevalence of delayed development scores based on the Griffiths Mental Development Scale (GMDS).

Results From 2009 to 2014, 1946 neonates were diagnosed with jaundice; 129 (6.6\%) had extreme SBR values during $\mathrm{NH}$ (extreme $\mathrm{NH})$. In this group, the median peak SBR was 430 (IQR 371-487) $\mu \mathrm{mol} / \mathrm{L}$ and the prevalence of $\mathrm{ABE}$ was $28.2 \%$. Extreme NH-related mortality was $10.9 \%$ (14/129). Median percentile GMDS general score of 37 survivors of extreme NH was poor: 11 (2-42). 'Performance', 'practical reasoning' and 'hearing and language' domains were most affected. Four (10.8\%) extreme NH survivors had normal development scores ( $\geq 50$ th centile). Two (5.4\%) developed the most severe form of kernicterus spectrum disorders.

Conclusion In this limited-resource setting, poor neonatal survival and neurodevelopmental outcomes, after extreme $\mathrm{NH}$, were high. Early identification and adequate treatment of $\mathrm{NH}$ where ET is not readily available are key to minimising the risk of extreme SBR values or neurological symptoms.

\section{(C) Author(s) (or their} employer(s)) 2020. Re-use permitted under CC BY. Published by BMJ.

For numbered affiliations see end of article.

Correspondence to Dr Verena llona Carrara; verena@shoklo-unit.com

\section{BACKGROUND}

In the most severe form of neonatal hyperbilirubinaemia $(\mathrm{NH})$, serum bilirubin (SBR) levels rise to extreme values, causing a lifethreatening condition that can lead to lifelong disability. ${ }^{1}$ Acute bilirubin encephalopathy (ABE) happens during the early phase of

\section{What is known about the subject?}

Extreme values of serum bilirubin (SBR) in the neonatal period can lead to death and long-term disability.

- Subtle disabilities and neurodevelopment delay might occur after recovery of neonata hyperbilirubinaemia.

- Low-income and middle-income countries bear the highest burden of disease but have limited access to diagnosis, treatment and supportive care for disability.

\section{What this study adds?}

A large majority of neonates with extreme SBR levels survive after on-site phototherapy but limited access to exchange transfusion.

- Long-term neurodevelopmental impairments, more pronounced in 'performance', 'reasoning skills' and 'language', affect survivors already bearing a heavy burden of chronic malnutrition and infections.

- Early recognition and adequate treatment are key to minimising the risk of extreme SBR values or neurological symptoms.

the disease, producing a vast array of neurological symptoms from mild and reversible to severe and irreversible. Its physiopathology is complex; however, prematurity, sepsis, glucose-6-phosphate dehydrogenase (G6PD) deficiency and $\mathrm{ABO} /$ rhesus incompatibility contribute to its development. ${ }^{2-5}$ Neurological sequelae might progress to kernicterus spectrum disorders (KSD) ranging from subtle to severe motor and/or auditory dysfunction, ${ }^{6}$ as well as language delay, attention disorders or lower executive function capacities. ${ }^{7}$

A review of the worldwide burden of severe NH published in 2017 reported an incidence of 251.3 per 10000 live births in Southeast Asia, 
the second highest after Africa. ${ }^{8}$ NH-related mortality is high: 1309 deaths per 100000 live births in $2016 .{ }^{9}$ Lowincome and middle-income countries bear the heaviest burden; two-thirds of deaths and severe sequelae occur in sub-Saharan Africa and South Asia; 3\%-20\% of survivors have normal long-term development. ${ }^{10-13}$

Most NH-related research follows the American Academy of Paediatrics guideline for neonates with an estimated gestational age (EGA) of $\geq 35$ weeks, in which SBR levels of $>25 \mathrm{mg} / \mathrm{dL}(428 \mu \mathrm{mol} / \mathrm{L})$ at age $>72$ hours are considered extreme and require exchange transfusion (ET).$^{14}$ The British National Institute for Health and Clinical Excellence (NICE) guideline proposes dynamic treatment thresholds based on postnatal age and varying with gestational ages from 23 to $\geq 38$ weeks. ${ }^{15}$ SBR values considered extreme therefore start as low as $13 \mathrm{mg} / \mathrm{dL}$ (230 $\mathrm{mmol} / \mathrm{L})$ for the youngest EGA. However, publications reporting outcomes of neonates treated following these dynamic treatment thresholds are still rare. ${ }^{16}$

The Shoklo Malaria Research Unit (SMRU) set up a special care baby unit (SCBU) in 2008 in Mae La, the most populous refugee camp along the Thailand-Myanmar border, to provide basic care for neonates including oxygen, intravenous antibiotics, nasogastric feeding, phototherapy and basic laboratory tests. ${ }^{17}$ Starting 2011 , another two SCBUs were set up in clinics for migrant populations. Care for neonates with $\mathrm{NH}$ improved over time following the implementation of standardised guidelines based on the NICE, ${ }^{15}$ systematic heel prick SBR measurements with onsite bilirubinometer (Pfaff Medical Bilimeters 2 and 3) and irradiance level optimisation with the introduction of light emitting diode (LED) phototherapy units. ${ }^{18}$ In live births from 2009 to 2014, NH prevalence was 18\%; $9 \%$ of these neonates had at least one SBR value exceeding the NICE threshold at which ET should be considered. ${ }^{18}$ ET is an intervention with potentially serious complications performed in an intensive care unit rather than in a primary health facility. ${ }^{19}{ }^{20}$ Due to the inherent difficulties of sending a refugee or migrant person to a specialised centre, only some neonates could be referred.

The objective of this review was to evaluate the immediate and long-term clinical and neurodevelopmental outcomes of children from a low-resource setting with SBR values rising to levels considered extreme.

\section{METHODS}

Three SMRU SCBU facilities contributed to the data in refugees (1 January 2009-31 December $2014^{17}$ ) and in migrants (1 January 2011-31 December 2014) (figure 1).

\section{Inclusion criteria}

Clinical charts of all liveborn singletons of $\geq 28$ weeks EGA without major congenital abnormalities born to mothers attending SMRU antenatal care admitted for a clinical diagnosis of jaundice were reviewed.

\section{Definitions}

For this analysis, NH with SBR values rising above levels that could justify ET, reported in the text as 'extreme NH', was defined as either (1) two consecutive SBR measurements above the ET threshold of the NICE guideline, (2) SBR levels rising faster than $8.5 \mu \mathrm{mol} / \mathrm{L} /$ hour in combination with one SBR measurement above the ET threshold or with clinical symptoms of ABE, or (3) a clinical diagnosis of ABE. ${ }^{15}$

In the absence of a scoring system for neurological symptoms, a simplified ABE definition was used and consisted of two or more symptoms mentioned in the chart ('quiet', 'sleepy', 'less sucking', 'irritable', 'lethargic', 'floppy', 'hypotonic', 'rigid', 'apnoea', 'seizure' and 'kernicterus') occurring within 2 days of the SBR peak. ${ }^{21}$

A computerised formula based on the NICE threshold graphs was used to select charts with SBR measurements fitting inclusion criteria. Clinical charts and originally drawn SBR trajectories were cross-checked to confirm eligibility. The first author and a paediatrician (LT) reviewed clinical charts for classifying available neurological symptoms, collecting ET referral and possible cause of death information.

Three available factors aggravating the risk of $\mathrm{ABE}$ other than prematurity (blood group $\mathrm{ABO}$ incompatibility, G6PD deficiency measured by fluorescent spot test $^{22}$ and neonatal sepsis) were extracted from computerised databases. Clinical diagnoses followed the local consensus-based SMRU neonatal guidelines. EGA of 35 weeks defined prematurity in this cohort as it is commonly used to assess the risk of extreme $\mathrm{NH}$ in the literature. ${ }^{312} 2324$ In this setting, most pregnancies have EGA calculated by ultrasound ${ }^{25}$ or by the best estimate of gestation available if there is late presentation to antenatal care. ${ }^{26}$ SBR and G6PD results were provided in near real time by locally trained, routinely quality controlled laboratory technicians.

\section{Neurodevelopmental assessment}

Children with extreme NH discharged alive from SCBU were traced using their parents address. For those consenting to participate, a one-off appointment was organised at the nearest SMRU clinic between November 2016 and March 2017. Medical history extracted from the child's medical booklet and clinical and neurological examination findings were reported on a standardised form. Maternal concern about the child's wellbeing was evaluated by asking whether the child had difficulty seeing, hearing, speaking, bathing, dressing or in terms of mobility, and reported as yes/no. The Griffiths Mental Development Scales-Extended Revised (GMDS-ER ${ }^{27}$ ) was used to assess neurocognitive development: it spanned the possible age distribution of this cohort and was familiar to the staff who could perform it in their own language. Test items were scored as 'pass' or 'fail' on six individual subscales, and percentile scores were derived from raw scores. A general neurodevelopment score EGA-adjusted was calculated per GMDS-ER 


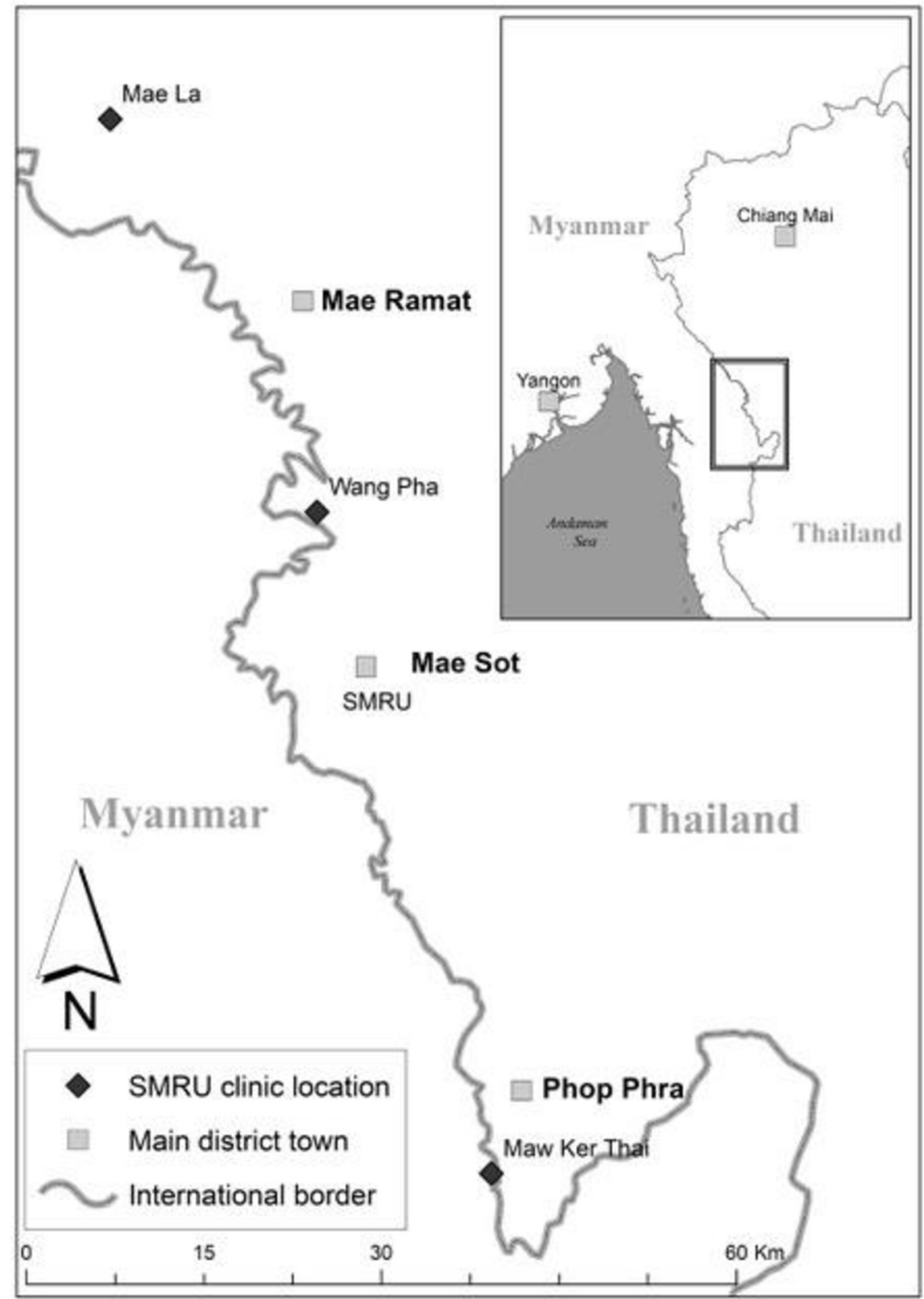

Figure 1 Location of the three SMRU clinics. The three clinics are Mae La clinic for refugees, Wang Pha clinic and Maw Ker Thai clinic for migrant population. The SMRU main office is located in Mae Sot town. Photo credit: Daniel Parker. SMRU, Shoklo Malaria Research Unit.

administration and analysis manuals and categorised as poor $(<10$ th percentile), delayed (10-49th percentiles) and normal (50th percentile or above). Visual function was assessed with the Cardiff cards for visual acuity and visual contrast. ${ }^{28}$ All tests were performed by local staff trained and regularly quality-controlled in clinical neurological examination and neurodevelopmental testing. ${ }^{29} 30$

\section{Statistics}

Analysis was performed using IBM SPSS Statistics for Macintosh V.24.0.0.1. Continuous data were described by their median and IQR and compared using the MannWhitney test. Categorical data were described by their proportion and compared with $\chi^{2}$ test, Fisher's exact test or $\chi^{2}$ test for trend as appropriate. Factors associated with
$\mathrm{ABE}$ were evaluated in univariate analysis and reported as OR with $95 \%$ CI.

\section{Patient and public involvement}

The Tak Province Border Community Ethics Advisory Board members representing the local community where the study took place reviewed the protocol and their input into the design of the project was taken into account.

\section{RESULTS}

Between 2009 and 2014, 13538 SMRU antenatal attendees gave birth at $\geq 28$ weeks' EGA to 12963 congenitally normal singletons; 2980 (23.0\%) neonates were hospitalised in the SCBU; 1946 were diagnosed with jaundice; 
2980 neonates $\geq 28$ weeks of gestation admitted to the SCBU between 2009-2014

1946 neonates admitted to the SCBU with clinical diagnosis of jaundice

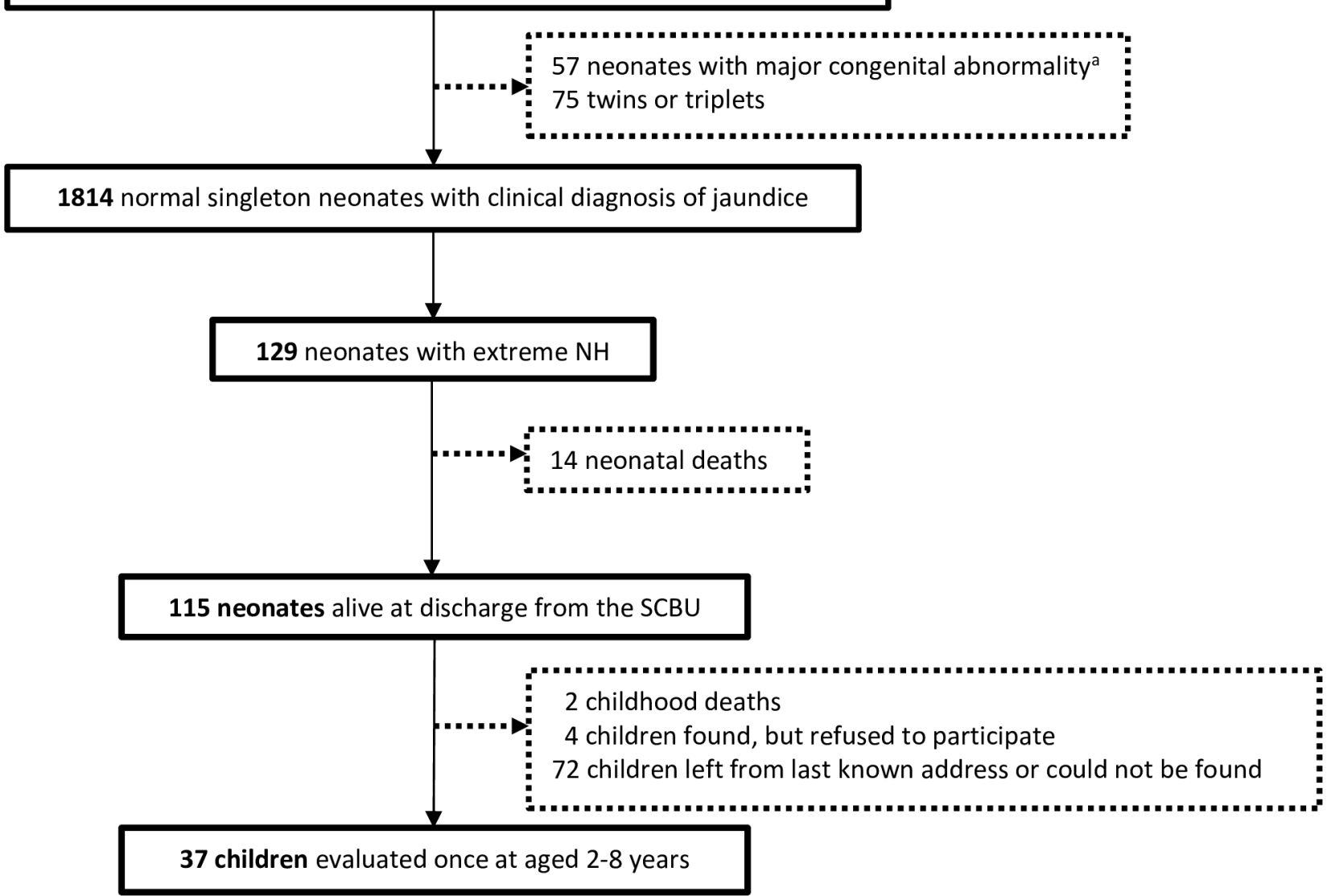

Figure 2 Flowchart representing the selection of neonates with extreme hyperbilirubinaemia (NH). ${ }^{a}$ Major congenital abnormality was defined as an abnormal finding on surface examination and/or auscultation of the heart and lungs reported in the medical chart and confirmed by a medical doctor. NH, neonatal hyperbilirubinaemia; SCBU, special care baby unit.

and 129 fulfilled the criteria of extreme NH (figure 2). Extreme $\mathrm{NH}$ incidence among neonates delivered in SMRU birthing units $(110 / 10398)$ was estimated at 105.8 per 10000 live births (95\% CI 87.0 to 127.4 ).

The general characteristics of the 129 neonates are summarised in table 1 . The majority $(70.5 \%)$ were from the refugee camp as expected given the longer data period; almost half $(46.5 \%)$ were born from primigravid mothers and $37(28.7 \%)$ were $<35$ weeks' EGA. Sepsis was clinically diagnosed prior to onset of $\mathrm{NH}$ in $26.4 \%$ of hospitalisations.

The median (IQR) value of the SBR peak was 430 (371487) $\mu \mathrm{mol} / \mathrm{L}$ and occurred at a median of 97 (68-136) hours of life. Five neonates were referred; four successfully underwent ET; one died of sepsis without confirmation of ET being attempted.

\section{Mortality and ABE}

Extreme $\mathrm{NH}$ case fatality rate was $10.9 \%$, giving a mortality rate of 108.1 per 100000 live births (95\% CI
59.1 to 181.4 ); neurological symptoms compatible with ABE were reported in $28.2 \%$ of the clinical charts of neonates not transferred for ET (35/124). All deaths occurred among neonates with ABE.

The odds of developing ABE was higher among neonates born <35 weeks' EGA $(41.6 \%$, 15/36, vs $22.7 \%$, 20/88, OR 2.4 (95\% CI 1.1 to 5.7$)$ ) or treated for clinical sepsis prior to onset of NH $(51.6 \%, 16 / 31$, vs $20.4 \%$, $19 / 93$, OR 4.2 (95\% CI 1.8 to 9.9$)$ ) but not for those with blood group $\mathrm{ABO}$ incompatibility or G6PD deficiency.

\section{Long-term outcomes}

Thirty-seven children survivors of extreme $\mathrm{NH}$ were present for neurodevelopmental assessment, including three who received ET. Their neonatal and maternal characteristics were similar to those of the 78 unavailable or untraceable children (table 2 ).

Clinical, visual, neurological and neurodevelopmental outcomes are presented in table 3. While none of the children seen were acutely ill, $30.6 \%$ reported more than 
Table 1 Characteristics of neonatal period of 129 neonates with extreme $\mathrm{NH}$

\section{Characteristics}

\begin{tabular}{|c|c|c|}
\hline \multicolumn{2}{|l|}{ Coracteristics } & \multirow{2}{*}{$22(17.0)$} \\
\hline Year of admission & 2009 & \\
\hline & 2010 & $26(20.2)$ \\
\hline & 2011 & $12(9.3)$ \\
\hline & 2012 & $24(18.6)$ \\
\hline & 2013 & $27(20.9)$ \\
\hline & 2014 & $18(14.0)$ \\
\hline \multirow[t]{2}{*}{ Status } & Refugee & $91(70.5)$ \\
\hline & Migrant & $38(29.5)$ \\
\hline Gender (male) & & $75(58.1)$ \\
\hline Gestational age $\geq 35$ weeks $^{*}$ & & $92(71.3)$ \\
\hline \multirow[t]{3}{*}{ Place of delivery } & SMRU clinic & $110(85.3)$ \\
\hline & Tertiary hospital & $4(3.1)$ \\
\hline & Home & $15(11.6)$ \\
\hline Birth weight $(\mathrm{kg})(\mathrm{n}=125)$, median $(\mathrm{IQR}) \dagger$ & & $2.53(1.96-2.90)$ \\
\hline Small for gestational age $(n=125) \ddagger$ & & $23(18.4)$ \\
\hline Poor start to life§ & & $15(11.6)$ \\
\hline Mother primigravid & & $60(46.5)$ \\
\hline Normal vaginal delivery & & $114(88.4)$ \\
\hline Age at admission (days), median (IQR) & & $1(0-4)$ \\
\hline Duration of admission (days), median (IQR) & & $6(4-11)$ \\
\hline \multirow[t]{3}{*}{ Extreme NH category } & Two SBRs above ET & $101(78.3)$ \\
\hline & Rapid SBR rise+ABE signs & $27(20.9)$ \\
\hline & Clinical diagnosis of $\mathrm{ABE}$ & $1(0.8)$ \\
\hline Duration of phototherapy (hours), median (IQR) & & $76(48-124)$ \\
\hline Peak SBR ( $\mu \mathrm{mol} / \mathrm{L})$, median (IQR) & & $430(371-487)$ \\
\hline Age at peak SBR (hours), median (IQR) & & $97(68-136)$ \\
\hline Peak SBR at $<72$ hours of life & & $41(31.8)$ \\
\hline Underwent ET & & $4(3.1)$ \\
\hline Potential blood group ABO incompatibility** $(n=126)$ & & $24(19.0)$ \\
\hline G6PD-deficient male†† ( $n=75)$ & & $33(44.0)$ \\
\hline Polycythaemiał‡ & & $10(7.8)$ \\
\hline Birth trauma (visible bruising after birth recorded in the chart) & & $6(4.7)$ \\
\hline Clinical diagnosis of sepsis prior to onset of $\mathrm{NH} \S \S$ & & $34(26.4)$ \\
\hline
\end{tabular}

Data shown in number and percentage (\%) unless stated otherwise.

${ }^{*}$ Gestational age: based on first trimester ultrasound. ${ }^{25}$

†Birthweight: weight measurement considered valid if done in the first 72 hours of life.

¥Small for gestational age: defined as birth weight below the 10th percentile for gestational age and sex, calculated with the INTERGROWTH-

$21 \mathrm{~s}^{t}$ newborn size application tool (https://intergrowth21.tghn.org).

$\S$ Poor start to life: Apgar score $<7$ at $5 \mathrm{~min}$ and/or clinical suspicion of meconium aspiration and/or resuscitation at birth with at least five inflation breaths.

IExtreme NH categories: (1) two consecutive SBR measurements above the ET threshold of the NICE guideline, (2) SBR levels rising faster than $8.5 \mu \mathrm{mol} / \mathrm{L} / \mathrm{hour}$ in combination with one SBR measurement above the ET threshold or with clinical symptoms of acute bilirubin encepalopathy, and (3) a clinical diagnosis of ABE.

**Potential blood group ABO incompatibility: mother-foetus pairs with mother blood group 'O' and neonate blood group 'A' or 'B'. Rhesus factor and Coombs test were not available.

††Diagnosed by G6PD fluorescent test. ${ }^{22}$

¥¥Polycythaemia: two consecutive haematocrit values $>70 \%$ from a capillary sample or a diagnosis of polycythaemia recorded in the clinical chart.

$\S \S$ Clinical diagnosis of sepsis: sepsis reported as clinical diagnosis treated at least 5 days by intravenous antibiotics (blood culture confirmations were not available).

ABE, acute bilirubin encepalopathy; ET, exchange transfusion; G6PD, glucose-6-phosphate dehydrogenase; NH, neonatal hyperbilirubinaemia; NICE, National Institute for Health and Clinical Excellence; SBR, serum bilirubin; SMRU, Shoklo Malaria Research Unit. 
Table 2 Neonatal and maternal characteristics of extreme NH survivors present for neurodevelopmental assessment $(n=37)$ and those unavailable or untraceable $(n=78)$

\begin{tabular}{llll}
\hline Characteristics & $\begin{array}{l}\text { Present } \\
(\mathbf{n = 3 7 )}\end{array}$ & $\begin{array}{l}\text { Untraceable } \\
(\mathbf{n = 7 8 )}\end{array}$ & P value \\
\hline Gender (male) & $20(54.1)$ & $47(60.3)$ & 0.529 \\
\hline Year of birth (2012-2014) & $24(64.9)$ & $41(52.6)$ & 0.214 \\
\hline Refugee status & $23(62.1)$ & $54(69.2)$ & 0.452 \\
\hline First born & $20(54.1)$ & $44(56.4)$ & 0.812 \\
\hline EGA (weeks+days), median (IQR) & $37+4(34+5-38+3)$ & $37+4(35+1-38+6)$ & 0.578 \\
\hline EGA<35 weeks & $11(29.7)$ & $18(23.1)$ & 0.443 \\
\hline Small for gestational age & $5 / 35(14.3)$ & $14 / 75(18.7)$ & 0.571 \\
\hline Normal vaginal delivery & $31(83.8)$ & $70(89.7)$ & 0.361 \\
\hline Days in SCBU, median (IQR) & $8(5-16)$ & $7(4-13)$ & 0.201 \\
\hline Clinical diagnosis of sepsis prior to onset of NH & $8(21.6)$ & $18(23.1)$ & 0.862 \\
\hline Maternal age, median (IQR) & $24(20-30)$ & $22(19-28)$ & 0.391 \\
\hline Maternal illiteracy & $9 / 27(33.3)$ & $23 / 57(40.4)$ & 0.536 \\
\hline Maternal smoking & $7(18.9)$ & $11(14.1)$ & 0.525 \\
\hline
\end{tabular}

Data shown in number and percentage (\%) unless stated otherwise.

EGA, estimated gestational age; $\mathrm{NH}$, neonatal hyperbilirubinaemia; SCBU, special care baby unit.

one hospitalisation per year. The proportion of chronic malnutrition was high ( $40.5 \%$ of children stunted), and half of the participants had teeth damage with black staining or cavities. Abnormal visual examination was reported in $21.6 \%$ of children, mostly related to poor acuity. None of the children wore glasses. Overall, children born $<35$ weeks' EGA were reporting similar, if not slightly better clinical condition than children born term (differences non-significant).

Two survivors of extreme NH (5.4\%), both born at term, had an abnormal neurological examination compatible with a severe form of KSD: one with upward and downward gaze paralysis, dystonia and suspected left-sided hearing loss, the other with cerebral palsy. Their general neurodevelopment score was below the first percentile.

The median (IQR) percentile summary score of all GMDS-ER subscales was low (11 (2-42)), with 16 children $(43.2 \%)$ scoring below the 10th centile; scores were particularly poor in the subscales 'hearing and language', 'performance' and 'practical reasoning' (table 3). Differences observed between gestational age groups did not reach significance level; however, children born $<35$ weeks' EGA tended to obtain similar or higher scores than those born term. Four survivors of extreme $\mathrm{NH}$ $(10.8 \%)$ had a general percentile score above the $50^{\text {th }}$ centile, the highest score reaching the $82^{\text {th }}$ centile.

\section{DISCUSSION}

This study reports the immediate outcomes and longterm consequences of extreme $\mathrm{NH}$ in a limited-resource setting. Reflecting on the burden estimates for South-East Asia of 251.3 per 10000 live live births and the worldwide 1309 deaths per 100000 live births presented in the publications by Slusher ${ }^{8}$ and Olusanya ${ }^{9}$ both the incidence of extreme $\mathrm{NH}$ and its mortality rate during this 6 year evaluation are reassuringly low. This likely reflects the benefit of onsite SBR measurements and thus an early phototherapy initiation. The case fatality rate among neonates with extreme $\mathrm{NH}$ treated with phototherapy was similar to that described in tertiary paediatric referral hospitals in Myanmar. ${ }^{31}$

Clinical symptoms compatible with ABE were present in $28.2 \%$ of neonates, a proportion within the range reported in the literature ${ }^{332}$ but with deleterious consequences: all deaths occurred among neonates with $\mathrm{ABE}$ and of the two children with severe neurological impairment at least one was suspected to be $\mathrm{ABE}$ sequelae. Routine recording of clinical symptoms using a system such as the Bilirubin-Induced Neurological Dysfunction scales might help capture earlier neurological abnormalities and enhance closer monitoring alongside the use of dynamic treatment thresholds. ${ }^{21}$ This, however, could only be of added benefit if timely referral can be organised as well. As a first step, emphasising the need for systematic SBR monitoring, ensuring regular feeds during phototherapy, and verifying that adequate irradiance levels are provided, are achievable recommendations to minimise ABE risk. ${ }^{18} 33$

The one-off neurodevelopmental evaluation results ranged from severely impaired to developmentally normal consistent with current published rates. ${ }^{7}$ Test performance was particularly poor in domains related to language and reasoning skills, both plausibly affected by bilirubin neurotoxicity. ${ }^{6} 74$ Reassuringly, survivors of extreme $\mathrm{NH}<35$ weeks' EGA performed similarly or even better than those born term despite a higher risk of developing ABE.

Whether children with severe KSD retain a normal intelligence is debated. ${ }^{1635}$ As an anecdotal case, and 
Table 3 Long-term clinical, visual, neurological and neurodevelopmental outcomes of 37 survivors of extreme NH by gestational age category

\begin{tabular}{|c|c|c|c|}
\hline & $\begin{array}{l}\text { Gestational age }<35 \text { weeks } \\
(n=11)\end{array}$ & $\begin{array}{l}\text { Gestational age } \\
\geq 35 \text { weeks }(n=26)\end{array}$ & $\begin{array}{l}\text { All } \\
(n=37)\end{array}$ \\
\hline \multicolumn{4}{|l|}{ General characteristics } \\
\hline Age at testing (months) (median, IQR) & $62.5(36.5-93.0)$ & $43.8(34-64.5)$ & $51.0(35-81)$ \\
\hline Age $<4$ years old & $3(27.3)$ & $13(50.0)$ & $16(43.2)$ \\
\hline Gender (male) & $4(36.4)$ & $16(61.5)$ & $20(54.1)$ \\
\hline First born & $8(72.7)$ & $12(46.2)$ & $20(54.1)$ \\
\hline Received exchange transfusion & 0 & $3(11.5)$ & $3(8.1)$ \\
\hline Refugee status & $7(63.6)$ & $16(61.5)$ & $23(62.2)$ \\
\hline \multicolumn{4}{|l|}{ Clinical outcomes } \\
\hline Being unwell ${ }^{\star}$ & $3(27.3)$ & $8 / 25(32.0)$ & $11 / 36(30.6)$ \\
\hline Stunting† & $2(18.2)$ & $13(50.0)$ & $15(40.5)$ \\
\hline Teeth abnormality $\ddagger$ & $5 / 10(50.0)$ & $13 / 26(50.0)$ & $18 / 36(50.0)$ \\
\hline \multicolumn{4}{|l|}{ Visual and neurological outcomes } \\
\hline Visual examination abnormality§ & $1(9.1)$ & 7 (26.9) & $8(21.6)$ \\
\hline Neurological exam abnormality & 0 & $2(7.7)$ & $2(5.4)$ \\
\hline \multicolumn{4}{|l|}{ Neurodevelopmental outcomes } \\
\hline $\begin{array}{l}\text { Maternal perception of child's development: mother has } \\
\text { worries }\end{array}$ & $3(27.3)$ & $3(11.5)$ & $6(16.2)$ \\
\hline General GMDS percentile score, median (IQR) & $32(11-42)$ & $7(<1-42)$ & $11(2-42)$ \\
\hline Poor development ( $<10$ th centile) & $2(18.2)$ & $14(53.8)$ & $16(43.2)$ \\
\hline Delayed development (10-49th centile) & $7(63.6)$ & $10(38.5)$ & $17(46.0)$ \\
\hline Normal development ( $\geq 50$ th centile) & $2(18.2)$ & $2(7.7)$ & $4(10.8)$ \\
\hline Locomotion score, median (IQR) & $34(19-59)$ & $12(3-58)$ & $19(5-58)$ \\
\hline Personal and social score, median (IQR) & $23(8-94)$ & $32(15-64)$ & $26(11-64)$ \\
\hline Hearing and language score, median (IQR) & $12(6-57)$ & $11(<1-33)$ & $11(<1-33)$ \\
\hline Eye and hand coordination score, median (IQR) & $27(4-46)$ & $14(4-48)$ & $22(4-46)$ \\
\hline Performance score, median (IQR) & $26(6-80)$ & $3(<1-19)$ & $4(<1-40)$ \\
\hline Practical reasoning score, median (IQR) ${ }^{\star \star}$ & $18(2-51)$ & $11(1-43)$ & $14(2-49)$ \\
\hline
\end{tabular}

Data shown in number and percentage (\%).

*Being unwell: more than one hospitalisation per year or presence of a chronic disease as reported in the child's medical booklet. †Stunting: height-for-age z-scores below -2 as calculated with WHO Child Growth Standards (http://www.who.int/childgrowth/en/). $\ddagger$ Teeth abnormality: judgement of medical staff looking for the presence of black staining or cavities, confirmed by first author through photographic records.

§Visual examination abnormality: unconjugated eye movement and Cardiff's binocular visual contrast and visual acuity lower than the normal values by age categories (instructions manual for the Cardiff Acuity Test).

INeurological examination abnormality: judgement of the medical staff after general observation while moving, and evaluating tone, strength and sensibility, coordination, gait and posture, and reflexes.

${ }^{* *}$ One child had an adjusted age of $<2$ years old when tested; therefore, practical reasoning was available for only 10 preterm children. EGA, estimated gestational age; GMDS, Griffiths Mental Development Scale; NH, neonatal hyperbilirubinaemia; SCBU, special care baby unit.

even though it was not possible to measure intelligence, the single child with features compatible with classic kernicterus appeared frustrated with not being able to complete the proposed tasks.

The clinical findings of this study confirm the harsh environment in which the children try to thrive: a high burden of chronic malnutrition, infections, and poor visual and dental care. ${ }^{36-40}$ Moderate to severe delay in development after surviving extreme $\mathrm{NH}$ will add to an already heavy burden and to the worries of families. In such a precarious socioeconomic environment, caregivers of neonates with extreme $\mathrm{NH}$ should be encouraged to attend a baby clinic to actively identify and treat nutritional and clinical problems influencing growth and development, if this is available.

This study's results might not be extrapolated elsewhere due to its limitations. ABE symptoms thought to represent abnormal neurological conditions were extracted from the daily medical notes and the lack of a systematic checklist means that the proportion of $\mathrm{ABE}$ might have 
been underestimated. The absence of regular follow-up after discharge from the SCBU limited the search for survivors 2-8 years later to those families who remained in the area and possibly underestimated the mortality rate while overestimating the proportion of children with severe delayed development if one assumes the family would remain closest to what little healthcare services are available. Neurodevelopmental tests were performed by locally trained staff fluent in local languages rather than by specialists, and cultural and environmental factors could have influenced the overall poor test outcomes. Nevertheless, all children came from the same environment; school exposure was generally limited; and the health staff was routinely quality controlled to report the pass/fail items as per test guideline and was unaware of the final scoring.

\section{CONCLUSION}

Extreme $\mathrm{NH}$ is a serious neonatal health issue in this limited-resource setting and possibly contributes to delayed development of children already vulnerable to a heavy burden of chronic malnutrition and infections. Early identification and adequate treatment of $\mathrm{NH}$ where ET is not readily available are key to minimising the risk of extreme SBR values or neurological symptoms; additional efforts are necessary to encourage parents to attend the baby clinic to identify and treat early concomitant health and nutritional problems.

\section{Author affiliations}

${ }^{1}$ Shoklo Malaria Research Unit, Mahidol-0xford Tropical Medicine Research Unit, Faculty of Tropical Medicine, Mahidol University, Mae Sot, Thailand

${ }^{2}$ University Medical Center, University of Groningen, Groningen, Netherlands ${ }^{3}$ Neonatology-Pediatrics Department, Hôpital Erasme, Université Libre de Bruxelles, Bruxelles, Belgium

${ }^{4}$ Faculty of Tropical Medicine, Mahidol-0xford Tropical Medicine Research Unit, Mahidol University, Bangkok, Thailand

${ }^{5}$ IRD-INSERM-SESSTIM, Aix-Marseille Université, Marseille, France

${ }^{6}$ Paediatric Gastroenterology, University Medical Center Groningen, Groningen, Netherlands

${ }^{7}$ Cambodia-0xford Medical Research Unit, Angkor Hospital for Children, Siem Reap, Cambodia

${ }^{8}$ Centre for Tropical Medicine and Global Health, Nuffield Department of Medicine, University of Oxford, Oxford, UK

${ }^{9}$ Department of Medicine, Swiss Tropical and Public Health Institute, Basel, Switzerland

Acknowledgements We thank all the SMRU staff and the parents and children who were willing to join in the study. Thanks to Stepping Stones physiotherapy for caring for the disabled children.

Contributors EMNW, LT and VIC conceived and designed the study, coordinated the study and supervised the research teams; PFvR, CT, FN and RMcG contributed to the study design. MCD, AAN, WS and SK performed all the neurodevelopment tests and clinical examinations. TN developed the data collection and provided the quality control. EMNW, LT and VIC led the analysis and interpretation of the data with JL leading the statistical analysis. All authors supported the preparation and later critically reviewed the manuscript, including in its final approval, and had full access to all of the data (including statistical reports and tables) in the study and can take responsibility for the integrity of the data and the accuracy of the data analysis.

Funding Shoklo Malaria Research Unit is part of the Mahidol-0xford University Tropical Medicine Research Program at the Faculty of Tropical Medicine, Mahidol
University, Bangkok, Thailand, and supported by the Wellcome Trust of Great Britain. The funders had no role in study design, data collection and analysis, decision to publish or preparation of the manuscript. LT was supported by a PhD grant from 'The Belgian Kids' Fund for Pediatric Research.

Map disclaimer The depiction of boundaries on the map(s) in this article does not imply the expression of any opinion whatsoever on the part of BMJ (or any member of its group) concerning the legal status of any country, territory, jurisdiction or area or of its authorities. The map(s) are provided without any warranty of any kind, either express or implied.

Competing interests None declared.

Patient consent for publication Not required.

Ethics approval The study was approved by the Oxford Tropical Research Ethics Committee (OXTREC 5113-16) and the ethics committee of the Faculty of Tropical Medicine of the Mahidol University (TMEC 16-071). The Tak Province Border Community Ethics Advisory Board representing the local community reviewed and accepted the study protocol (TCAB-01/REV/2016).

Provenance and peer review Not commissioned; externally peer reviewed.

Data availability statement Datasets used for this analysis are available from the corresponding author upon reasonable request.

Open access This is an open access article distributed in accordance with the Creative Commons Attribution 4.0 Unported (CC BY 4.0) license, which permits others to copy, redistribute, remix, transform and build upon this work for any purpose, provided the original work is properly cited, a link to the licence is given, and indication of whether changes were made. See: https://creativecommons.org/ licenses/by/4.0/.

\section{ORCID iDs}

Eva Maria Nadine Wouda http://orcid.org/0000-0002-7192-3422

Verena llona Carrara http://orcid.org/0000-0002-2758-0872

\section{REFERENCES}

1 Bhutani VK, Johnson-Hamerman L. The clinical syndrome of bilirubin-induced neurologic dysfunction. Semin Fetal Neonatal Med 2015;20:6-13.

2 Bhutani VK, Wong RJ, Stevenson DK. Hyperbilirubinemia in preterm neonates. Clin Perinatol 2016;43:215-32.

3 Gamaleldin R, Iskander I, Seoud I, et al. Risk factors for neurotoxicity in newborns with severe neonatal hyperbilirubinemia. Pediatrics 2011;128:e925-31.

4 Watchko JF, Tiribelli C. Bilirubin-induced neurologic damage-mechanisms and management approaches. $N$ Engl J Med 2013;369:2021-30.

5 Weng Y-H, Chiu Y-W, Cheng S-W, et al. Risk assessment for adverse outcome in term and late preterm neonates with bilirubin values of $20 \mathrm{mg} / \mathrm{dL}$ or more. Am J Perinatol 2011;28:405-12.

6 Le Pichon J-B, Riordan SM, Watchko J, et al. The neurological sequelae of neonatal hyperbilirubinemia: definitions, diagnosis and treatment of the kernicterus spectrum disorders (KSDs). Curr Pediatr Rev 2017;13:199-209.

7 Wusthoff CJ, Loe IM. Impact of bilirubin-induced neurologic dysfunction on neurodevelopmental outcomes. Semin Fetal Neonatal Med 2015;20:52-7.

8 Slusher TM, Zamora TG, Appiah D, et al. Burden of severe neonatal jaundice: a systematic review and meta-analysis. BMJ Paediatr Open 2017;1:e000105.

9 Olusanya BO, Teeple S, Kassebaum NJ. The contribution of neonata jaundice to global child mortality: findings from the GBD 2016 study. Pediatrics 2018;141. doi:10.1542/peds.2017-1471. [Epub ahead of print: 0501 2018].

10 Bhutani VK, Johnson L. Kernicterus in late preterm infants cared for as term healthy infants. Semin Perinatol 2006;30:89-97.

11 Bhutani VK, Zipursky A, Blencowe H, et al. Neonatal hyperbilirubinemia and rhesus disease of the newborn: incidence and impairment estimates for 2010 at regional and global levels. Pediatr Res 2013;74 Suppl 1:86-100.

12 Mukhopadhyay K, Chowdhary G, Singh P, et al. Neurodevelopmental outcome of acute bilirubin encephalopathy. J Trop Pediatr 2010;56:333-6.

13 Sgro M, Campbell DM, Kandasamy S, et al. Incidence of chronic bilirubin encephalopathy in Canada, 2007-2008. Pediatrics 2012;130:e886-90.

14 American Academy of Pediatrics Subcommittee on Hyperbilirubinemia. Management of hyperbilirubinemia in the 
newborn infant 35 or more weeks of gestation. Pediatrics 2004;114:297-316.

15 NICE. Neonatal jaundice: clinical guideline. London: national collaborating centre for women's and children's health, 2010. Available: https://www.nice.org.uk/guidance/cg98/evidence/fullguideline-245411821 [Accessed 30 December 2019].

16 Olusanya BO, Emokpae AA. Use of transcutaneous bilirubin to determine the need for phototherapy in resource-limited settings. Neonatology 2017;111:324-30.

17 Turner C, Carrara V, Aye Mya Thein N, et al. Neonatal intensive care in a Karen refugee cAMP: a 4 year descriptive study. PLoS One 2013;8:e72721.

18 Thielemans L, Trip-Hoving M, Landier J, et al. Indirect neonatal hyperbilirubinemia in hospitalized neonates on the Thai-Myanmar border: a review of neonatal medical records from 2009 to 2014 BMC Pediatr 2018;18:190.

19 Mabogunje CA, Olaifa SM, Olusanya BO. Facility-based constraints to exchange transfusions for neonatal hyperbilirubinemia in resource-limited settings. World J Clin Pediatr 2016;5:182-90.

20 Sanpavat S. Exchange transfusion and its morbidity in tenyear period at King Chulalongkorn Hospital. J Med Assoc Thai 2005;88:588-92.

21 Radmacher PG, Groves FD, Owa JA, et al. A modified bilirubininduced neurologic dysfunction (BIND-M) algorithm is useful in evaluating severity of jaundice in a resource-limited setting. BMC Pediatr 2015;15:28.

22 Thielemans L, Gornsawun G, Hanboonkunupakarn B, et al. Diagnostic performances of the fluorescent spot test for G6PD deficiency in newborns along the Thailand-Myanmar border: a cohort study. Wellcome Open Res 2018;3:1.

23 Ebbesen $\mathrm{F}$, Andersson $\mathrm{C}$, Verder $\mathrm{H}$, et al. Extreme hyperbilirubinaemia in term and near-term infants in Denmark. Acta Paediatr 2005;94:59-64.

24 Kuzniewicz MW, Wickremasinghe AC, Wu YW, et al. Incidence, etiology, and outcomes of hazardous hyperbilirubinemia in newborns. Pediatrics 2014;134:504-9.

25 Rijken MJ, Mulder EJH, Papageorghiou AT, et al. Quality of ultrasound biometry obtained by local health workers in a refugee cAMP on the Thai-Burmese border. Ultrasound Obstet Gynecol 2012;40:151-7.

26 Moore KA, Simpson JA, Thomas KH, et al. Estimating gestational age in late Presenters to antenatal care in a resourcelimited setting on the Thai-Myanmar border. PLoS One 2015; 10:e0131025.
27 Barnett AL, Guzzetta A, Mercuri E, et al. Can the Griffiths scales predict neuromotor and perceptual-motor impairment in term infants with neonatal encephalopathy? Arch Dis Child 2004;89:637-43.

28 Adoh TO, Woodhouse JM, Oduwaiye KA. The Cardiff test: a new visual acuity test for toddlers and children with intellectual impairment. A preliminary report. Optom Vis Sci 1992;69:427-32.

29 Janet S, Carrara VI, Simpson JA, et al. Early neonatal mortality and neurological outcomes of neonatal resuscitation in a resource-limited setting on the Thailand-Myanmar border: a descriptive study. PLOS One 2018;13:e0190419.

30 Haataja L, McGready R, Arunjerdja R, et al. A new approach for neurological evaluation of infants in resource-poor settings. Ann Trop Paediatr 2002;22:355-68.

31 Arnolda G, Nwe HM, Trevisanuto D, et al. Risk factors for acute bilirubin encephalopathy on admission to two Myanmar national paediatric hospitals. Matern Health Neonatol Perinatol 2015;1:22.

32 Salas AA, Mazzi E. Exchange transfusion in infants with extreme hyperbilirubinemia: an experience from a developing country. Acta Paediatr 2008;97:754-8.

33 Olusanya BO, Ogunlesi TA, Kumar P, et al. Management of latepreterm and term infants with hyperbilirubinaemia in resourceconstrained settings. BMC Pediatr 2015;15:39.

34 Shapiro SM, Popelka GR. Auditory impairment in infants at risk for bilirubin-induced neurologic dysfunction. Semin Perinatol 2011;35:162-70.

35 Ip S, Chung M, Kulig J, et al. An evidence-based review of important issues concerning neonatal hyperbilirubinemia. Pediatrics 2004:114:e130-53.

36 Boel M, Carrara VI, Rijken M, et al. Complex interactions between soil-transmitted helminths and malaria in pregnant women on the Thai-Burmese border. PLoS Negl Trop Dis 2010;4:e887.

37 Kemmer TM, Bovill ME, Kongsomboon W, et al. Iron deficiency is unacceptably high in refugee children from Burma. $J$ Nutr 2003;133:4143-9.

38 Swe ZY, Narksawat K, Chansatitporn N. Dental caries status and oral health behavior among Myanmar migrant workers in MAE Sot district, Tak Province, Thailand. Myanmar Dental Journal 2016.

39 Turner C, Turner P, Carrara V, et al. High rates of pneumonia in children under two years of age in a South East Asian refugee population. PLoS One 2013;8:e54026.

40 UNICEF. Situation analysis of children with disabilities in the Republic of the Union of Myanmar. Myanmar: Yangon: UNICEF, 2016. https:// reliefweb.int/sites/reliefweb.int/files/resources/Full_report_in_ English_0.pdf 